\title{
Test of Host Sanction Hypothesis in Soybean Plants Co-inoculated with Nitrogen Fixing and Non-fixing Bradyrhizobium japonicum
}

\author{
Diana E. Marco ${ }^{1^{*}}$, Chouhra Talbi ${ }^{2}$ and Eulogio J. Bedmar ${ }^{3}$ \\ ${ }^{1}$ Faculty of Agricultural Sciences, National University of Córdoba and CONICET, University Campus \\ CC 509, 5000 Córdoba, Organic Production Area, Argentina. \\ ${ }^{2}$ Genomics Sciences Center, UNAM, Avenida Universidad 1001, Cuernavaca, Morelos, 62290, \\ México. \\ ${ }^{3}$ Department of Soil Microbiology and Symbiotic Systems, Zaidín Experimental Station, CSIC State \\ Agency, Granada, Spain.
}

\section{Authors' contributions}

This work was carried out in collaboration between all authors. Authors DEM and EJB designed the study, author DEM performed the experiments, statistical analysis, and wrote the manuscript. Author CT helped with protocols and lab work. All authors read and approved the final manuscript. All authors read and approved the final manuscript.

Article Information

DOI: $10.9734 / J S R R / 2015 / 15510$ Editor(s):

(1) Mario A. Pagnotta, Department of Science and Technologies for Agriculture, Forestry, Nature and Energy (DAFNE), Tuscia University, Italy. Reviewers:

(1) Elouaer Mohamed Aymen, Department of Horticulture Sciences, High Institute of Agronomy, Tunisia. (2) Reda Abdel-Zaher Abdel-Aziz, Agricultural Microbiology Department, National Research Centre, Egypt. (3) Anonymous, Japan Complete Peer review History: http://www.sciencedomain.org/review-history.php?iid=963\&id=22\&aid=8081

\section{Original Research Article}

Received $30^{\text {th }}$ November 2014 Accepted $26^{\text {th }}$ January 2015 Published $7^{\text {th }}$ February 2015

\section{ABSTRACT}

Aims: We tested the proposed mechanism for potential sanctions, that the plant would reduce viability of non-fixing rhizobia inside nodules, performing viable Bradyrhizobium japonicum counts from co-occupied and single-occupied nodules in co-inoculated soybean plants.

Study Design: Plants were co-inoculated with two strains of $B$. japonicum, a highly efficient nitrogen fixing wild-type strain BJD321, and the non-fixing, nifH mutant derivative A3, to produce co-occupied nodules as well as single-occupied nodules. Strain A3 lacks nitrogenase activity but shows similar infection and nodule formation levels respect to the wild-type. As the strains used are equivalent in competitive and nodulation abilities and only differ in the nitrogen fixation ability (by nitrogenase inactivation), and share the same plant, root and even nodule, we can assert that the 
mechanism being tested is plant host sanction, and no other proposed mechanisms like partner choice.

Place and Duration of Study: Nitrogen Metabolism Lab, Department of Soil Microbiology and Symbiotic Systems at Zaidín Experimental Station (CSIC State Agency, Granada, Spain). 20102011.

Methodology: Axenic seedlings of soybean (Glycine max) cultivar Williams were inoculated with 2 $\mathrm{ml}$ of bacterial suspension of BJD321 or A3 strains, alone or in 1:1 mixture and supplied with sterilized $\mathrm{N}$ free nutrient solution. Four weeks after inoculation plants of each inoculation treatment (BJD321 + A3, BJD321 only, A3 only) were harvested, nodules were counted and weighed and plated to determine rhizobial strain occupation and population. In the aerial part of plants, determinations of weight, $\mathrm{N}$ and $\mathrm{C}$ content were done.

Results: Co-inoculated plants and plants only inoculated with the BJD321 strain showed a similar nitrogen fixation since they did not differ in dry weight, total $\mathrm{N}$ content and total $\mathrm{C}$ content. Plants with different inoculation treatments (BJD321 + A3, BJD321 only and A3 only) did not differ in nodule number. In co-inoculated plants, nodule occupation did not differ from the expected among strains (about 33\% BJD321 + A3, BJD321 only and A3 only), and the weight of nodules occupied by both strains, BJD321 or A3 alone did not differ. In co-inoculated plants rhizobial viability did not differ between BJD321 and A3 strains, either comparing co-occupied nodules or single-occupied nodules. Nodule size and CFU of rhizobia inside nodules were not correlated, either in coinoculated plants or plants inoculated with BJD321 strain alone.

Conclusion: We can conclude that in the soybean- $B$. japonicum system, plants facing the presence of fixing and non-fixing rhizobial strains do not sanction cheating and can perform as well as plants inoculated with the fixing strain alone.

Keywords: Legume-rhizobia mutualism; plant host sanction; nodule co-occupation.

\section{INTRODUCTION}

The evolutionary stability of mutualisms has been for long a theoretical puzzle, since the existence of defective, cheating mutualistic partners is well known [1]. To answer the question of how can cooperation be maintained if some partners may seek only self-benefit, different mechanisms have been proposed that could defend mutualisms from cheating [2,3,4]. Despite theoretical explanations, cheating and exploitative strategies appear to be amply spread in nature $[5,6]$, including legume-rhizobia mutualism [7]. In this interaction, bacteria known as rhizobia from the soil infect meristematic cells of the plant's root through a sophisticated signaling mechanism between mutualistic partners and a new organ is formed, termed nodule. Nodules may be occupied by a one or more strains, and may grow until a certain size is reached (determinate nodules, e.g. soybean), or keep a meristematic growth zone (indeterminate nodules, e.g. pea). Inside the nodule rhizobia reproduce and differentiate into bacteroids able to fix atmospheric $\mathrm{N}_{2}$ for plant utilization. Reciprocally, the plant provides carbohydrated compounds to rhizobia in the nodules. Following nodule senescence, surviving bacteroids (in determinate nodules) or undifferentiated bacteria (in indeterminate nodules) are released into the soil as free-living rhizobia, where the infection process restarts the next growing season involving strain competence for nodulation [8].

The presence of low $\mathrm{N}_{2}$-fixing or even ineffective rhizobia cheating strains nodulating legumes has been recognized for long in agricultural practices $[9,10]$. A specific mechanism, termed plant host sanction, has been proposed as a stabilizing force defending plant mutualism from cheating rhizobia [11]. The plant would penalize cheating rhizobia by reducing their survival inside the nodule through a reduction in nodule permeability to $\mathrm{O}_{2}$, necessary for bacteroid respiration, and/or causing premature nodule senescence [11,12]. Kiers et al. [13,14] reported a decrease in viability of Bradyrhizobium japonicum recovered from soybean nodules when $\mathrm{N}_{2}$-fixing $B$. japonicum were 'forced' to cheat soybean plants by replacing normal, $\mathrm{N}_{2}$ containing atmosphere by a gas mixture $\left(\mathrm{Ar}: \mathrm{O}_{2}\right)$, and pronounced this result as an example of plant sanction. However, in an alternative approach with half-roots of split-root soybean plants inoculated with fixing and non-fixing $B$. japonicum strains, Marco et al. [15] did not find a decrease in rhizobia viability nor premature nodule senescence in nodules occupied by the non-fixing strain. Thus, in our opinion, the issue of the soundness of the plant-host sanction hypothesis in the soybean- $B$. japonicum mutualism is still an open question. 
An interesting scenario relevant to the plant host sanction hypothesis deals with the potential responses of plant hosts facing cheating rhizobia co-occupying nodules with fixing strains. In field, about 20 to $32 \%$ of total nodules can be cooccupied by different rhizobial strains in artificial inoculations [16,17]. Following the ideas of Denison [11], effects of co-occupation of nodules by non-fixing rhizobia would be diluted by fixing rhizobia occupying the same nodule, thus not favouring plant sanctions. To our knowledge, experiments dealing with plant host sanctions in soybean plants with co-occupied nodules by $B$. japonicum strains with different fixation abilities have not been performed yet.

Here, we tested the proposed mechanism for potential sanctions, that the plant would reduce viability of non-fixing rhizobia inside nodules, performing viable $B$. japonicum counts from cooccupied and single-occupied nodules in coinoculated soybean plants. Plants were coinoculated with two strains of $B$. japonicum, a highly efficient $\mathrm{N}_{2}$-fixing strain BJD321 [18], and the non-fixing, nifH mutant $A 3$ [19], to produce co-occupied nodules as well as single-occupied nodules. Strain A3 lacks nitrogenase activity but shows similar infection and nodule formation levels respect to the wild-type [19]. As the strains used are equivalent in competitive and nodulation abilities and only differ in the nitrogen fixation ability (by nitrogenase inactivation), and share the same plant, root and even nodule, we can assert that the mechanism being tested is plant host sanction [11], and no other proposed mechanisms like partner choice, where the host establish preferential association with rhizobia in the steps previous to nitrogen fixation $[4,20]$. Comparisons relevant to the plant host-sanction hypothesis will be performed, between fixing and non-fixing strains co-occupying the same nodule and fixing and non-fixing strains inside singleoccupied nodules.

\section{MATERIAL AND METHODS}

\subsection{Bacterial strains and Inoculum Preparation}

The $\mathrm{N}_{2}$-fixing strain Bradyrhizobium japonicum BJD321 [19] (hereafter "fix+") and the non- $\mathrm{N}_{2}$ fixing $B$. japonicum strain A3 (hereafter "fix-") [21] were used in this study. Strain A3 lacks nitrogenase activity but shows similar infection and nodule formation levels respect to USDA110spc4 [21] and BJD321 [19]. Cells were routinely grown in peptone salts yeast (PSY) medium [22] supplemented with the respective antibiotics $\left(\mu \mathrm{g} / \mathrm{ml}, \mathrm{Spc}^{100}\right.$ and $\mathrm{Tc}^{200}$ for BJD321 and $\mathrm{Sm}^{100}$ and $\mathrm{Km}^{100}$ for $\mathrm{A} 3$ ), and a growth curve for each strain was adjusted by calculating the regression between cell count as colony-forming units (CFU) in plated dilutions and optical density (OD) at $600 \mathrm{~nm}$ (Supplementary material, Fig. S1). For the 1:1 mixed inocula, cells of each strain were collected at OD suited to render the same cell concentration (around $10^{9} \mathrm{cell} / \mathrm{ml}$ ), washed to eliminate the antibiotics, resuspended in PSY medium and subsequently mixed.

\subsection{Plant Experimental Setting}

Seeds of soybean (Glycine max) cultivar Williams were surface sterilized using absolute alcohol and $\mathrm{H}_{2} \mathrm{O}_{2}$, germinated and sowed in Leonard jars assemblies containing sterilized vermiculite. Seedlings of each jar were inoculated with $2 \mathrm{ml}$ of bacterial suspension of BJD321 or A3 strains, alone or in 1:1 mixture. A scheme of the inoculation treatments and the expected nodule strain occupation in each treatment is given in Fig. 1.

Jars were periodically supplied with sterile- $\mathrm{N}$ free nutrient solution [23], thus the only $\mathrm{N}$ source for plants would be the $\mathrm{N}_{2}$ fixed by rhizobia, depending on the inoculation treatment. Plants were placed in a growth chamber with $16 \mathrm{~h}$ and $600 \mathrm{\mu Em}^{-2} \mathrm{~s}^{-1}$ photosynthetically active radiation at $25^{\circ} \mathrm{C}$, and $8 \mathrm{~h}$ darkness at $18^{\circ} \mathrm{C}$. Control uninoculated plants showed no nodulation. Four weeks after inoculation 6 plants of each inoculation treatment (BJD321 + A3, BJD321 only, A3 only), were harvested and all the nodules in each plant were counted. In the remaining plants, nodules of each plant in coinoculated treatment (BJD321 + A3) and only inoculated with BJD321 were collected. To avoid biases from different nodule sizes and ages, all nodules of each plant (6 plants/treatment) were independently weighed and used immediately for determination of nodule occupancy.

Plants with all nodules occupied by non-fixing rhizobia were not able of maintaining good vegetative conditions as plants with co-occupied or exclusively occupied nodules with fixing rhizobia (Figs. S1a, b), and ultimately they died due to $\mathrm{N}$ starvation about 5 weeks after inoculation (Fig. S1c). This was expected since A3 only inoculated plants received no $\mathrm{N}$ at all. Thus, as the aerial part of A3 inoculated plants were in very bad state, determinations of dry weight, $\mathrm{N}$ and $\mathrm{C}$ content were done only in coinoculated plants and only BJD321 inoculated 
plants. For dry weight determinations plants (aerial part + roots) were oven-dried until constant weight was obtained. For $\mathrm{N}$ and $\mathrm{C}$ content determination oven-dried shoots were weighed and grounded in an IKA A11 basic analytical mill (Rose Scientific Ltd., Alberta, Canada). Subsamples of approximately $3 \mathrm{mg}$ were weighed and analysed for total $\mathrm{N}$ and $\mathrm{C}$ using an elemental analyser (EA1500 NC, Carlo Erba, Milan, Italy).

\subsection{Determination of Nodule Occupancy and Viable Rhizobial Counts}

Collected nodules from co-inoculated plants were individually surface-sterilized using $\mathrm{HgCl}_{2}(2.5 \%)$, manually crushed, homogenized and resuspended in a buffer containing $0.05 \mathrm{M}$ Tris$\mathrm{HCl}$ and $0.25 \mathrm{M}$ mannitol. Each crushed nodule was streaked on PSY plates supplemented with selective antibiotics depending on the strain to determine if the nodule was occupied by BJD321, A3 or both strains. To determine rhizobial viability, appropriate serial dilutions from another set of homogenized nodules were plated (three replicates per dilution) in PSY supplemented with selective antibiotics depending on the strain. Plates were incubated at $28^{\circ} \mathrm{C}$ for a week or until no further growth was detected, and colony-forming units (CFU) were counted. Determination of CFU was also made on nodules from plants inoculated with BJD321 strain only. Since soybean plants may compensate against ineffective nodulations by increasing effective nodule mass [24], CFU numbers from individual nodules were compared per nodule mass with Mann Whitney- $U$ test analysis on original, untransformed data. A total of 60 nodules were inspected.

\section{RESULTS}

Co-inoculated plants and plants only inoculated with the BJD321 strain showed a similar nitrogen fixation since they did not differ in dry weight, total $\mathrm{N}$ content and total $\mathrm{C}$ content (Table 1).

Plants with different inoculation treatments (BJD321 + A3, BJD321 only and A3 only) did not differ in nodule number, which ranged between 180 and 120 nodules per plant (Fisher's Exact Significance $=.135$, Median Test, $n=6$ ).

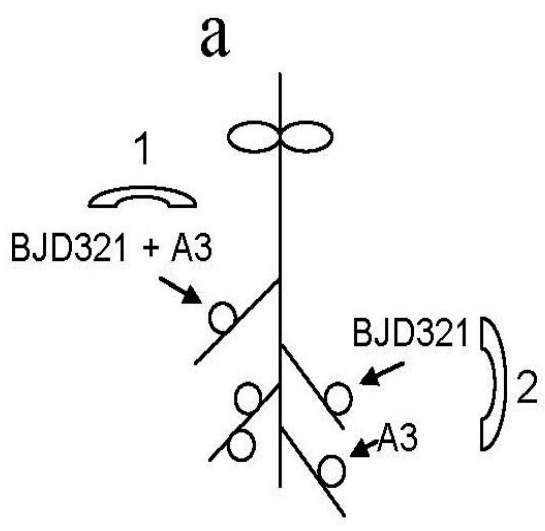

Co-inoculated plants

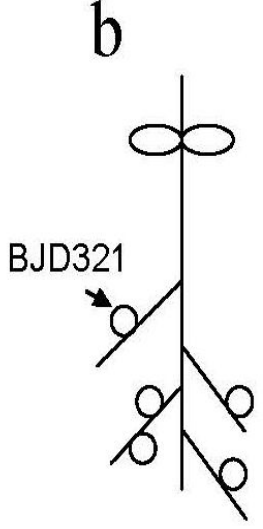

Only BJD321 inoculated plants

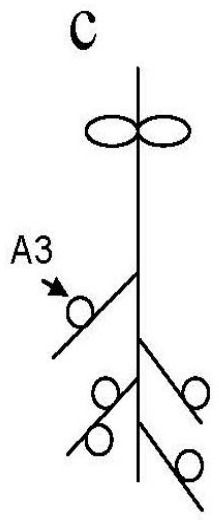

Only A3 inoculated plants

Fig. 1. Scheme of the inoculation treatments. Plants inoculated with a $1: 1$ mix of strains BJD321 (fix+) and A3 (fix-) (a) were expected to produce co-occupied nodules (BJD321+A3 strains), and single-occupied nodules (either by BJD321 only or A3 only). Plants inoculated with only BJD321 strain (fix+ control) (b) or with only A3 strain (fix-control) (c) were expected to produce nodules occupied only by either strain. Comparisons relevant to the plant host sanction hypothesis: between BJD321 and A3 strains inside the same nodule (1) and between BJD321 and A3 strains inside single nodules each (2) 
In co-inoculated plants, nodule occupation did not differ from the expected among strains $\left(x^{2}=\right.$ $4, P=.20, \mathrm{n}=6$ ) (Fig. 2).

In co-inoculated plants, weight of nodules occupied by both strains, BJD321 or A3 alone did not differ $\left(X^{2}=0.389, P=.82, \mathrm{n}=24\right)$ (Fig. 3). Weight of nodules occupied by both strains or BJD321 alone in co-inoculated plants did not differ from weight of nodules from plants only inoculated with BJD321 $\left(x^{2}=1.041, P=.60, \mathrm{n}=\right.$ 30).

Comparisons of number of CFU of rhizobia relevant to the plant host sanction hypothesis $(1$,
2 in Fig. 1, Table S1) showed that in coinoculated plants rhizobial viability did not differ between BJD321 and A3 strains, either comparing co-occupied nodules (comparison 1) (Mann Whitney- $U=40, P=.32$ ) or singleoccupied nodules (comparison 2) (Mann Whitney- $U=51, P=.10$ ). Viability of rhizobia from nodules of plants inoculated only with the BJD321 strain did not differ from viability of BDJ321 strain in co-occupied nodules but was significantly lower than BJD321 rhizobia from single-occupied nodules in co-inoculated plants (Mann Whitney-U $U$ = $17, \quad P=.01$ ) (Fig. 4, Table S2).

Table 1. Plant dry weight and $\mathrm{N}$ and $\mathrm{C}$ plant content ( $\mathrm{mg} \mathrm{g}-1$ dry weight) for inoculation treatments. Co-inoculated: plants inoculated with strains BJD321 (fix+) and A3 (fix-); Only BJD321: plants inoculated with strain BJD321 alone.

\begin{tabular}{|c|c|c|c|c|c|c|}
\hline & \multicolumn{2}{|c|}{ Plant dry weight (g) } & \multicolumn{2}{|c|}{ Plant $\mathrm{N}$ content $\left(\mathrm{mg} \mathrm{g}^{-1}\right)$} & \multicolumn{2}{|c|}{ Plant $C$ content $\left(\mathrm{mg} \mathrm{g}^{-1}\right)$} \\
\hline & Co-inoc. & Only BJD321 & Co-inoc. & Only BJD321 & Co-inoc. & Only BJD321 \\
\hline Median & 1.33 & 1.31 & 29.45 & 35.56 & 330.52 & 337.06 \\
\hline Range & 2.80 & 1.45 & 6.12 & 13.62 & 80.05 & 54.06 \\
\hline$P$-value & \multicolumn{2}{|l|}{1.000} & \multicolumn{2}{|c|}{.569} & \multicolumn{2}{|c|}{1.000} \\
\hline
\end{tabular}

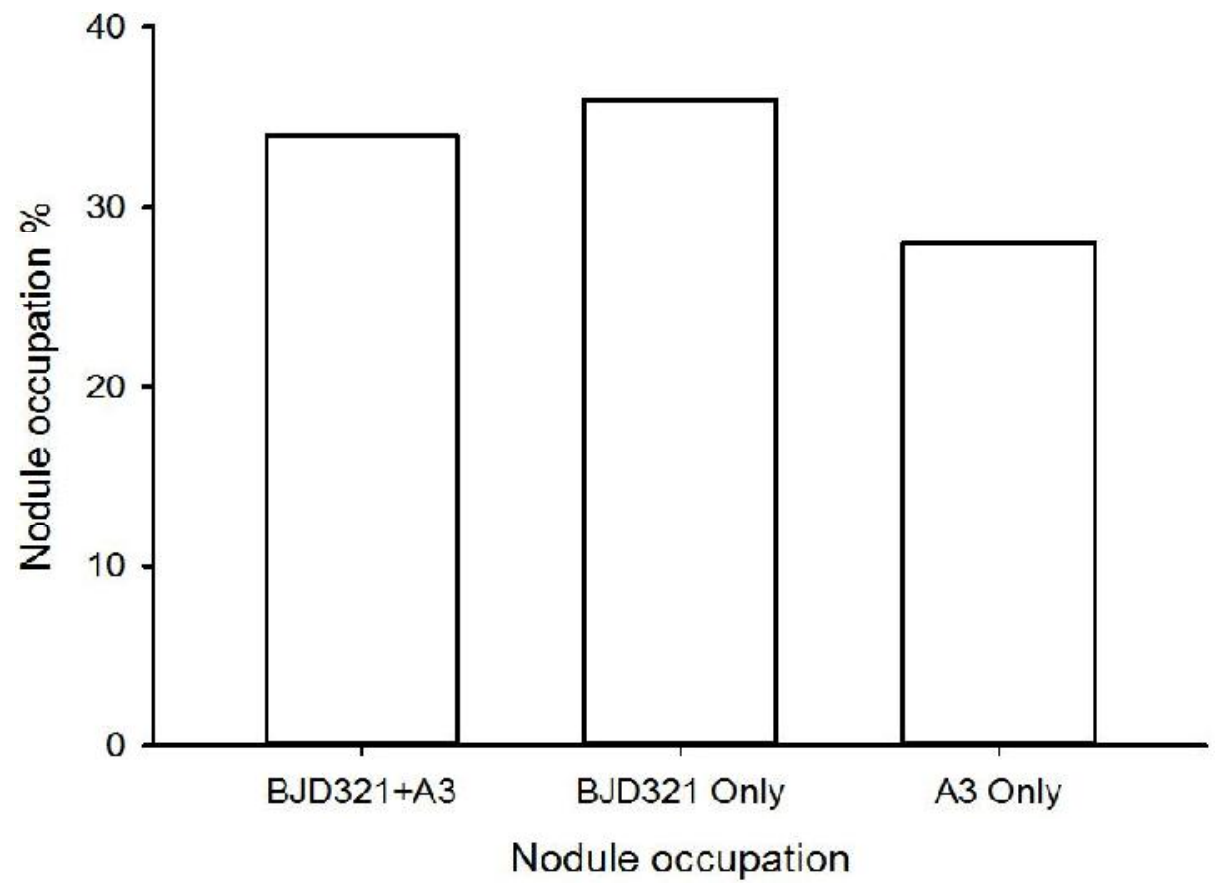

Fig. 2. Percentage of nodule occupation by BJD321 (fix+) and A3 (fix-) strains in co-inoculated plants. BJD321 + A3: nodules occupied by both strains, BDJ321-A3 Only: nodules occupied by either strain alone 


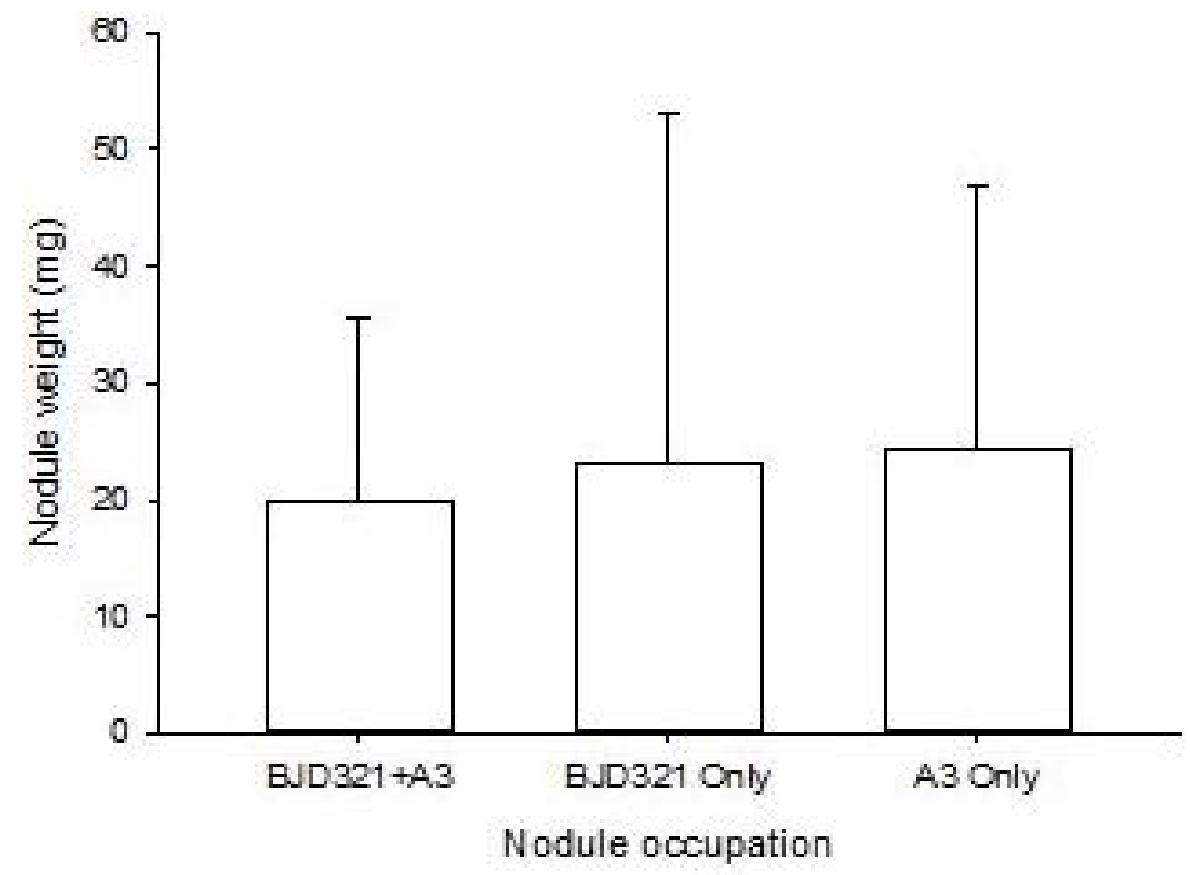

Fig. 3. Nodule weight in plants co-inoculated with BJD321 (fix+) and A3 (fix-) strains. BJD321 + A3: nodules occupied by both strains, BDJ321-A3 Only: nodules occupied by either strain alone. Bars are means \pm SD

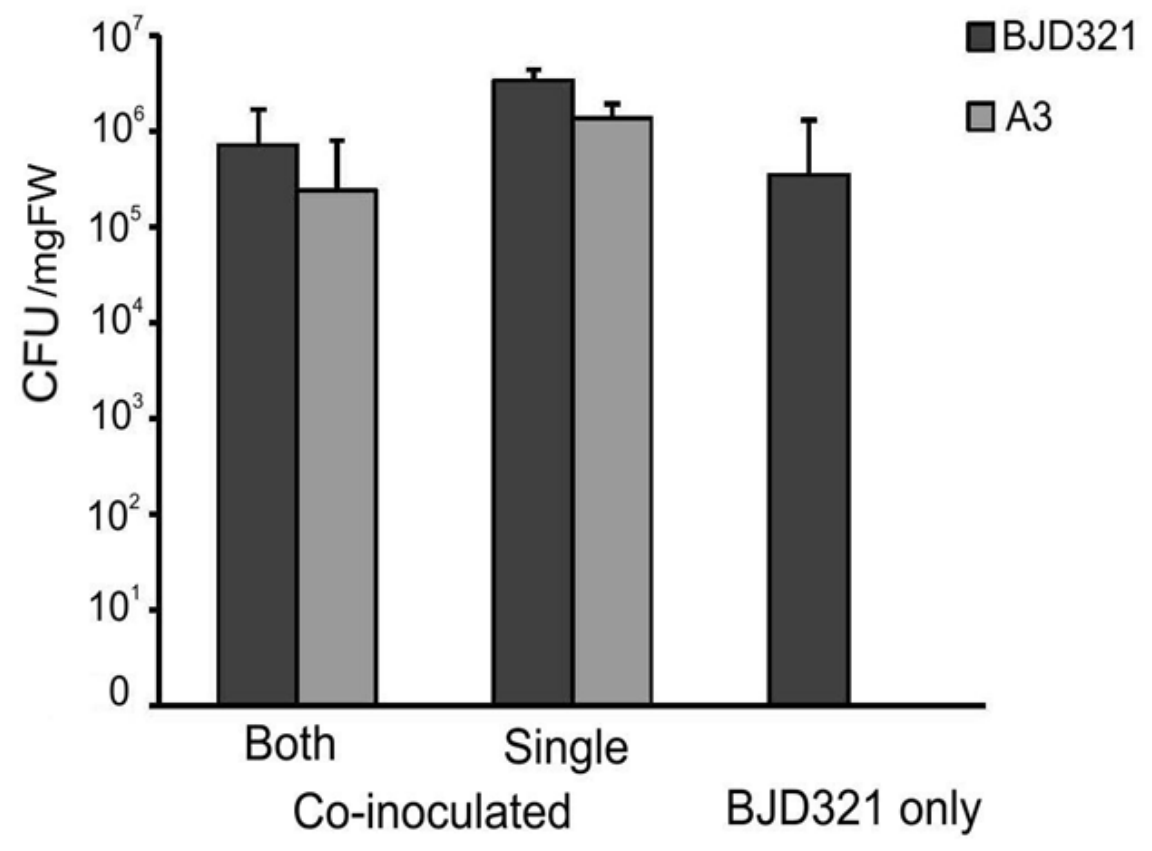

Fig. 4. Viability (as log number of CFU/mg nodule fresh weight) of rhizobia recovered from nodules with different inoculation treatments. Co-inoculated: plants inoculated with both strains BJD321 (fix+) and A3 (fix-): Both (co-occupied nodules with the two strains), Single (nodules occupied by one strain). BJD321 only: Plants inoculated with strain BJD321 alone 
Nodule size and number of CFU of rhizobia inside nodules were not correlated, either in coinoculated plants or plants inoculated with BJD321 strain alone (Table S2.)

\section{DISCUSSION}

We showed that fixing and non-fixing $B$. japonicum strains in co-occupied soybean nodules (comparison 1 in our experiment) did not differ in viability. Due to our experimental design, where the strains used are equivalent in competitive and nodulation abilities and only differ in the nitrogen fixation ability (by nitrogenase inactivation), and share the same plant, root and even nodule, we can confidently conclude that the cheating strain is not under host sanction. This is in agreement with the speculations from Denison [11], that in plants with determinate nodules host control at the level of the whole nodule would probably be less effective, since the plant can get some amount of fixed $\mathrm{N}_{2}$ from the effectively mutualistic rhizobia co-occupying the nodule. However, in the same plants, we found that viability of the fixing and the non-fixing strains occupying each a single nodule (comparison 2 of our experiment) also did not differ. Again, as the two strains differ only in nitrogenase activity, and share the plant and the root, we can conclude that the cheating strain is not undergoing host sanction. This finding contradicts previous results from Denison and colleagues [13,14,25], and does not provide support for the plant host sanction hypothesis, originally formulated for single-occupied nodules [11]. As we pointed out in a previous work [26] in our opinion the approach followed by Kiers $[13,14]$ does not really test a sanction from the plant to a true cheating rhizobium sharing the same plant with an effective strain. In addition, since exposure to an $\operatorname{Ar}: \mathrm{O}_{2}$ atmosphere per se reduces nodule $\mathrm{O}_{2}$ concentration in soybean nodules due to decrease in $\mathrm{O}_{2}$ nodule permeability through a not yet entirely understood mechanism [27,28,29], the experiments from Kiers et al. and Oono et al. $[13,14,25]$, could be in fact testing the effect of the $\mathrm{Ar}: \mathrm{O}_{2}$ treatment on nodules. Further doubts about the suitability of the $\mathrm{Ar}: \mathrm{O}_{2}$ experiments from Denison and colleagues to effectively test the plant-host sanction hypothesis have been raised by Frederickson [30]. On the other hand, and using an experimental method avoiding potentially confounding effects like the $\mathrm{Ar}: \mathrm{O}_{2}$ treatment, our present results are in agreement with our previous finding of no sanctions towards a cheating $B$. japonicum strain in the soybean split-root experiment mentioned earlier [26]. Other recent works on legume-rhizobia mutualisms using co-inoculated plants are given evidence that plants do not sanction cheating strains (in determinate nodules: Medicago truncatula-Sinorhizobium meliloti system: Gubry et al. [31]; Phaseolus vulgaris-Rhizobium etli system: Ling et al. [32]; in indeterminate nodules: M. truncatula and S. meliloti system: Heath and Tiffin [33]. On the other hand, works from Sachs et al. [34] and Regus et al. [35] on plants of Lotus strigosus (determinate nodules) co-inoculated with Bradyrhizobium strains with different fixation abilities, reported plant-host sanctions on the inefficient strains. However, our results are difficult to compare with those from Sachs and colleagues [34,35], since their experiments were designed to test both for partner choice and plant-host sanctions mechanisms of control over cheating rhizobial strains, and thus, the strains they used were not equivalent in infection and nodulation rates.

Although nodule co-occupation by strains with different fixation abilities is commonly found in the field [36] there are still few works specifically done considering this scenario [34,35]. As mentioned above, Sachs et al. and Regus et al. $[34,35]$ tested for partner choice as well as for plant-host sanctions in co-inoculated plants with strains differing in fixation ability as well as in infective and nodule formation abilities. To compare our results with those from Sachs and Regus [34,35], the most suitable situation would be the co-inoculation with an inefficient strain (strain 2) and a highly efficient one (strain 49) used in both works. Sachs et al. [34] found a low nodule co-occupation rate by strains 2 and 49 , and statistically smaller rhizobia populations of the inefficient strain 2 in one of the experiments (at 8 week harvest), but no detectable population of this strain earlier (at 5 weeks) (Table S4 in Sachs et al. [34]). These results are difficult to interpret in the light of the plant-host sanction mechanism due to the confounding effect of the already mentioned differences in infection ability. Results from Regus et al. [35] are even more difficult to compare with ours since none of the co-inoculations with strains 2 and 49 under zero $\mathrm{N}$ conditions did result in co-occupied nodules and moreover, there were none or very few nodules occupied by the inefficient strain (Table 1 in Regus et al. [35]). To our knowledge, there is only one published work with experimental conditions comparable to our present work, i.e., specifically testing plant-host sanctions in plants with determinate nodules and co-inoculating 
strains with equivalent nodulation rates but differing in fixation ability. Ling et al. [32] constructed Rhizobium etli mutants in nifA genes responsible for nitrogen fixation and coinoculated plants of Phaseolus vulgaris (determinate nodules) with these deficient mutants and a normally fixing wild type strain. Ling et al. [32] found a high number of cooccupied nodules and thus were able to confidently calculate the CFU number of nifA mutant and wild type strain in those nodules. The CFU ratio nifA/wild type inside co-occupied nodules was about 2 , showing a significant advantage of the cheating strain. Similarly to our results, Ling et al. [32] also found no differences in nodule weight and nitrogen fixation comparing nodules from co-inoculated plants and nodules containing the wild type strain alone. These latter results are interesting since it is usually accepted that nodules containing inefficient rhizobial strains are smaller than those occupied by fixing rhizobia, and this has been taken as a surrogate for rhizobial population sizes inside nodules [37]. However, Sachs et al. [37] did not find correlations between nodule size, rhizobial population and strain efficiency in $L$. strigosus inoculated with Bradyrhizobium strains with different fixation abilities, and even more, they found higher rhizobial populations inside nodules of plants inoculated with the inefficient strain 2. Simms et al. [20] reported smaller nodule size and in low efficient strains in a greenhouse experiment with Lupinus arboreus plants (indeterminate nodules) inoculated with Bradyrhizobium strains differing in nitrogen fixation. However, they determined nodule size and rhizobial populations in nodules from a different set of plants, nodule strain identity was not established, and this relationship was poorly correlated $\left(r^{2}=0.20, p<.01, n=33\right)$ [20]. Thus, to further clarify the relationship between nodule size, strain performance and rhizobial population size inside nodule more experiments should be done.

Our present results are in agreement with our previous findings from a mathematical model developed based on experiments with split-root soybean plants inoculated with fixing and cheating strains of $B$. japonicum [26]. Simulation results from our model showed that plant populations perform well even in the presence of cheating rhizobia without the need of incorporating any sanction against the cheater populations in the model, under the realistic assumption that plants can at least get some amount of fixed $\mathrm{N}_{2}$ from the effectively mutualistic rhizobia occupying some nodules. That scenario is equivalent to that of our present experiments, where plants, even hosting the cheating strain, can get a certain amount on nitrogen from the fixing strain from co-occupied and single-occupied nodules. As we shown, in co-inoculated plants the percentage of nodule occupation was about $30 \%$ for each occupation possibility (only BJD321, BJD321+A3, and A3 only). So, the plant is actually receiving $\mathrm{N}$ from the entire population of bacteroids in BJD321 occupied nodules plus the BJD321 population in BJD321+A3 occupied nodules. However, the $N$ fixed amount did not differ between co-inoculated and BJD321 only inoculated plants. Thus, it is tempting to think that the amount of fixed $\mathrm{N}$ in coinoculated plants should be about $50 \%$ of the $\mathrm{N}$ fixed in BJD321 only inoculated plants. However, it has been shown that there is a non-linear relationship between the amount of fixed $\mathrm{N}$ and the percentage (or number) of effective nodules in two soybean varieties inoculated with a mixture of effective and ineffective $R$. japonicum strains [24]. These authors found that a decline in both the proportion and number of effective nodules did not result in a proportional decline in $\mathrm{N}$ accumulation, and this was explained by a compensation in nodule mass (effective nodules were larger than ineffective ones). However, we did not find any compensation in nodule mass, since weight of nodules did not differ either among treatments in co-inoculated plants nor between co-inoculated plants and plants only inoculated with BJD321 strain. It may be that effectively a non-linear relationship between the amount of $\mathrm{N}$ fixed and the fraction of effective nodules (counting the mixed-occupied nodules as effective) exists but it does not depend on nodules mass compensation. This is supported by our finding of no correlation between nodule mass and number of CFU inside nodules in either in co-inoculated or only BJD321 inoculated plants.

Supporting our findings, recent reviews based on the empirical evidence show that in the legumerhizobia mutualism as well in other mutualistic systems is increasingly seen as is doubtful that cheaters select for host sanctions $[30,38]$.

\section{CONCLUSION}

Our experiments allowed us unequivocally test the plant-host sanction hypothesis in coinoculated plants with a normally fixing rhizobial strain and a non-fixing strain equivalent in nodulation rates. We did not find evidence of 
sanctions towards the non-fixing, cheating strain by measuring rhizobial populations either inside co-occupied nodules or in single-occupied nodules on the same plant and roots. Besides, we did not find evidence for poor performance in co-inoculated plants, either in aerial biomass or nitrogen content. We can conclude that in the soybean- $B$. japonicum system, plants facing the presence of fixing and non-fixing rhizobial strains do not sanction cheating and can perform as well as plants inoculated with the fixing strain alone.

\section{ACKNOWLEDGEMENTS}

This study was supported by ERDF-cofinanced grants P09-RNM-4746 from Consejería de Economía, Innovación y Ciencia (Junta de Andalucía, Spain). D.E.M. is a member of the National Research Council (CONICET, Argentina) and thanks the Ministry of Education of Spain for a Sabbatical Leave at Department of Soil Microbiology and Symbiotic Systems at Zaidín Experimental Station (Agencia CSIC, Granada, Spain). We thank the lab personal for general help, and Michael Götffert for $B$. japonicum BJD321 and Hans-Martin Fischer for A3 strains. We also thank anonymous reviewers for comments on the manuscript.

\section{COMPETING INTERESTS}

Authors have declared that no competing interests exist.

\section{REFERENCES}

1. Axelrod R, Hamilton WD. The evolution of cooperation. Science. 1981;211:13901396.

2. Frank SA. Foundations of social evolution. Princeton: Princeton Univ. Press; 1998.

3. Bull JJ, Rice WR. Distinguishing mechanisms for the evolution of cooperation. J Theor Biol. 1991;149:63-74.

4. Sachs JL, Mueller UG, Wilcox TP, Bull JJ. The evolution of cooperation. Quart Rev Biol. 2004;79:135-160.

5. Machado CA, Herre EA, McCafferty S, Bermingham E. Molecular phylogenies of fig pollinating and nonpollinating wasps and the implications for the origin and evolution of the fig-fig wasp mutualism. J Biogeogr. 1996;23:521-530.

6. Pellmyr O, Leebens-Mack J, Huth CJ. Non-mutualistic yucca moths and their evolutionary consequences. Nature. 1996;380:155-156.

7. Bronstein JL. The exploitation of mutualisms. Ecol Lett. 2001;4:277-287.

8. Hirsch P. Population dynamics of indigenous and genetically modified rhizobia in the field. New Phytol. 1996;133:159-171.

9. Amarger N. Competition for nodule formation between effective and ineffective strains of Rhizobium meliloti. Soil Biol Biochem. 1981;13:475-480.

10. Singleton PW, Tavares JW. Inoculation response of legumes in relation to the number and effectiveness of indigenous Rhizobium populations. Appl Environ Microbiol. 1986;51:1013-1018.

11. Denison RF. Legume sanctions and the evolution of symbiotic cooperation by Rhizobia. Amer Nat. 2000;156:567-576.

12. West SA, Kiers ET, Simms EL, Denison RF. Sanctions and mutualism stability: why do rhizobia fix nitrogen? Proc R Soc B. 2002;269:685-694.

13. Kiers ET, Rousseau RA, West SA, Denison RF. Host sanctions and the legume-rhizobium mutualism. Nature. 2003;425:78-81.

14. Kiers TE, Rousseau RA, Denison RF. Measured sanctions: legume hosts detect quantitative variation in rhizobium cooperation and punish accordingly. Evol Ecol Re. 2006;8:1077-1086.

15. Marco DE, Pérez-Arnedo R, Hidalgo-Perea A, Olivares J, Ruiz-Sainz JE, Sanjuán J. A mechanistic molecular test of the plantsanction hypothesis in legume-rhizobia mutualism. Acta Oecol. 2009;35:664-667.

16. Rolfe BG, Gresshoff PM. Rhizobium trifolii mutant interactions during the establishment of nodulation in white clover. Austr J Bio Sci. 1980;33:491-504.

17. Moawad M, Schmidt EL. Occurrence and nature of mixed infections in nodules of field-grown soybeans (Glycine max). Biol Fert Soils. 1987;5:112-114.

18. Zehner S, Schober G, Wenzel M, Lang K, Göttfert M. Expression of the Bradyrhizobium japonicum type III secretion system in legume nodules and analysis of the associated tts box promoter. MPMI. 2008;21:1087-1093.

19. Hahn M, Studer D. Competitiveness of a nif Bradyrhizobium japonicum mutant against the wild-type strain. FEMS Microbiol Lett. 1986;33:143-148. 
20. Simms EL, Taylor DL, Povich J, Shefferson RP, Sachs JL, Urbina M. An empirical test of partner choice mechanisms in a wild legume-rhizobium interaction. Proc R Soc B. 2006;273:7781.

21. Hahn M, Meyer L, Studer D, Regensburger $B$, Hennecke $H$. Insertion and deletion mutations within the nif region of Rhizobium japonicum. Plant Mol Biol. 1984;3:159-168.

22. Regensburger $B$, Hennecke $H$. RNA polymerase from Rhizobium japonicum. Arch Microbiol. 1983;135:103-109.

23. Rigaud J, Puppo A. Indole-3-acetic catabolism by soybean bacteroids. J Gen Microbiol. 1975;88:223-228.

24. Singleton PW, Stockinger KR. Compensation against ineffective nodulation in soybean. Crop Sci. 1983;23:69-72.

25. Oono R, Anderson C.G, Denison RF. Failure to fix nitrogen by non-reproductive symbiotic rhizobia triggers host sanctions that reduce fitness of their reproductive clonemates. Proc R Soc B. 2011;278:2698-2703.

26. Marco DE, Carbajal JP, Cannas SA, Pérez-Arnedo $R$, Hidalgo-Perea A, Olivares J, et al. An experimentally-based modelling exploration of the host-sanction hypothesis in legume-rhizobia mutualism. J Theor Biol. 2009;259:423-433.

27. King BJ, Layzell DB. Effect of increases in oxygen concentration during the argoninduced decline in nitrogenase activity in root nodules of soybean. Plant Physiol. 1991;96:376-381.

28. Diaz del Castillo L, Layzell DB. Drought stress, permeability to $\mathrm{O}_{2}$, diffusion, and the respiratory kinetics of soybean root nodules. Plant Physiol. 1995;107:11871194.
29. Wei H, Layzell DB. Adenylate-coupled ion movement. A mechanism for the control of nodule permeability to $\mathrm{O}_{2}$ diffusion. Plant Physiol. 2006;141:280-287.

30. Frederickson ME. Rethinking mutualism stability: Cheaters and the Evolution of Sanctions. Quart Rev Biol. 2013;88:269295.

31. Gubry-Rangin C, Garcia M, Béna G. Partner choice in Medicago truncatulaSinorhizobium symbiosis. Proc R Soc B. 2010;277:1947-1951.

32. Ling J, Zheng $\mathrm{H}$, Katzianer DS, Wang $\mathrm{H}$, Zhong Z, Zhu J. Applying reversible mutations of nodulation and nitrogenfixation genes to study social cheating in Rhizobium etli-legume interaction. PloS One. 2013;8:e70138.

33. Heath KD, Tiffin P. Stabilizing mechanisms in a legume-rhizobium mutualism. Evolution. 2009;63:652-662.

34. Sachs JL, Russell JE, Lii YE, Black KC, Lopez G, Patil AS. Host control over infection and proliferation of a cheater symbiont. J Evol Biol. 2010;23:1919-1927.

35. Regus JU, Gano KA, Hollowell AC, Sachs JL. Efficiency of partner choice and sanctions in Lotus is not altered by nitrogen fertilization. Proc $R$ Soc $B$. 2014;281:20132587.

36. Moawad H, El-Din SB, Abdel-Aziz R. Improvement of biological nitrogen fixation in Egyptian winter legumes through better management of Rhizobium. Plant Soil. 1998;204:95-106.

37. Sachs JL, Ehinger MO, Simms EL. Origins of cheating and loss of symbiosis in wild Bradyrhizobium. J Evol Biol. 2010;23:1075-1089.

38. Friesen ML. Widespread fitness alignment in the legume-rhizobium symbiosis. New Phytol. 2012;194:1096-1111.

(C) 2015 Marco et al.; This is an Open Access article distributed under the terms of the Creative Commons Attribution License (http://creativecommons.org/licenses/by/4.0), which permits unrestricted use, distribution, and reproduction in any medium, provided the original work is properly cited.

Peer-review history:

The peer review history for this paper can be accessed here: http://www. sciencedomain.org/review-history.php?iid=963\&id=22\&aid=8081 